\title{
Análise comparativa de técnicas de filtragem espacial em área rural na região do Médio Alto Uruguai - RS
}

\author{
Eliara Marin Piazza', Lílian Gonçalvez Mariano², Rafaela Badinelli Hummel’3, \\ Roselene Marostega Felker ${ }^{4}$, Franciele Coghetto ${ }^{5}$, Maureen de Moraes Stefanello ${ }^{6}$ \\ 123456 Universidade Federal de Santa Maria
}

\section{Resumo}

A filtragem de imagens é uma técnica utilizada para melhorar a qualidade de imagens digitais, através da incorporação de algoritmos, os quais permitem eliminar regiões indesejáveis, oriundas de métodos de aquisição ou de condições inadequadas de captura, que acabam por introduzir algum tipo de ruído nas imagens. Dessa forma, objetiva-se avaliar comparativamente diferentes filtros existentes, a fim de agregar conhecimentos à cerca do processo de filtragem espacial, já que estudos deste cunho ainda são escassos na literatura. Foram utilizadas imagem referente à zona rural do município de Frederico Westphalen, obtidas do satélite Landsat-5/TM, bandas $3(0,63$ - 0,69 $\mu \mathrm{m}), 4(0,76$ - 0,90 $\mu \mathrm{m})$ e $5(1,55-1,75 \mu \mathrm{m})$ do ano de 1985 , previamente georreferenciadas que serviram de base para o registro das imagens. Observou-se que os processos de filtragem utilizados demonstraram que a eficiência dos filtros varia de acordo com o alvo, alterando o contraste, brilho e estrutura, sendo importante no processo de entendimento da deterioração causada na imagem original.

Palavras-chave: Frederico Westphalen; imagem espacial; Landsat-5/TM.

\begin{abstract}
The image filtering is a technique used to improve the quality of digital images through the incorporation of algorithms, which allow to eliminate unwanted regions, derived from procurement methods or inadequate capture that eventually introduce some kind of noise in images. Thus, the objective is to comparatively evaluate different existing filters in order to add to the knowledge about the process of spatial filtering, since studies of this nature are still scarce in the literature. Image were used regarding the rural municipality of Frederico Westphalen, Landsat-5/TM Imager bands 3 ( 0.63 to $0.69 \mu \mathrm{m}), 4(0.76$ to $0.90 \mu \mathrm{m})$ and $5(1,55$ to $1.75 \mu \mathrm{m})$ of the year 1985 , previously georeferenced which formed the basis for the registration of the images. It was observed that filtering processes used to demonstrate the efficiency of the filter varies with the target, changing the contrast, brightness and structure are important in the understanding of deterioration caused the original image.
\end{abstract}

Keywords: S Frederico Westphalen; spatial image; Landsat-5/TM. 


\section{INTRODUÇÃO}

Com o desenvolvimento de modernas tecnologias espaciais, tornou-se possível conhecer a terra através da aquisição de imagens da sua superfície por meio de sensores remotos. Os dados gerados por estes sensores têm-se demonstrado excelentes aliados na elaboração de diagnósticos de implicações ambientais, econômicas, sociais, políticas e culturais em relação à ocupação dos espaços geográficos (GONÇALVES, 2005).

No entanto, todas as imagens geradas através destas tecnologias, possuem limites entre áreas com diferentes respostas em relação à energia eletromagnética, dificultando a interpretação de feições com frequências específicas.

A fim de amenizar os erros de interpretação de imagens oriundas de sensores remotos e aperfeiçoar a qualidade da aparência da distribuição espacial das informações, técnicas de filtragem espacial de frequências são empregadas. Essas técnicas têm como objetivo principal realçar seletivamente as feições de alta, média ou baixa frequência que compõem as imagens de sensoriamento remoto.

De acordo com Müller e Daronco (2000) técnicas de filtragem são transformações da imagem pixel a pixel, que não dependem apenas do nível de cinza de um determinado pixel, mas também do valor dos níveis de cinza dos pixels vizinhos, o que faz com que a filtragem espacial, seja uma das técnicas mais utilizadas para melhorar a qualidade de uma imagem digital. Isso se deve ao fato de serem incorporados algoritmos que permitem eliminar regiões indesejáveis, oriundas de métodos de aquisição ou de condições inadequadas de capturada, que acabam por introduzir algum tipo de ruído na imagem. Com isso, o ruído atribui à imagem um aspecto granuloso que dificulta a discriminação dos alvos, tanto por métodos visuais quanto por algoritmos de segmentação e classificação (SOLBO E ELTOFT, 2008).

Gonzalez e Woods (1993) apontam que a filtragem de uma imagem pode ser considerada como a principal operação de manipulação de características espaciais. Dessa forma, a utilização de filtros, em busca da melhoria da qualidade da imagem, preservação da textura e redução do ruído em questão, são cada vez mais comuns.

No entanto, apesar da filtragem ser uma das principais operações de manipulação e de muitos filtros terem sido desenvolvidos, a comparação entre eles varia de acordo com o que o intérprete busca identificar. Dessa forma, muitas metodologias foram propostas, cada uma visando avaliar um determinado parâmetro. Neste contexto, o principal objetivo do trabalho foi avaliar comparativamente os diferentes filtros existentes, a fim de agregar conhecimentos a cerca da filtragem espacial, já que estudos deste cunho ainda são escassos na literatura.

\section{METODOLOGIA}

Para realização do estudo foi utilizada imagem referente à zona rural do município de Frederico Westphalen, localizada nas coordenadas $53^{\circ} 25^{\prime} 59.26^{\prime \prime} \mathrm{O}$ e $27^{\circ} 23^{\prime} 44.40^{\prime \prime} \mathrm{S}$. A referida imagem foi obtida do satélite Landsat-5/TM, bandas $3(0,63-0,69 \mu \mathrm{m}), 4(0,76-0,90 \mu \mathrm{m}) \mathrm{e}$ $5(1,55-1,75 \mu \mathrm{m})$ do ano de 1985 , previamente georreferenciadas que serviram de base para $o$ registro das imagens.

O processamento da mesma, ocorreu no Laboratório de Geoprocessamento da Universidade Federal de Santa Maria, Campus de Frederico Westphalen, onde foi utilizado o aplicativo SPRING 5.0, no qual se testou as técnicas de filtragem espacial linear e não linear.

Para a filtragem linear utilizou-se um filtro do tipo passa-baixa com máscara de $5 \times 5$, na banda do Infravermelho próximo. Em sequência foi usado o mesmo tipo de filtragem, ou seja, passa-baixa com máscara de $5 \times 5$, mas com banda correspondente ao vermelho.

Posteriormente para finalizar o processamento das imagens ocorreu a filtragem não linear do tipo mediana na banda do Infravermelho próximo, com máscaras diferenciadas, uma $3 \times 3$, $5 \times 5$, e outra $7 \times 7$.

\section{RESULTADOS E DISCUSSÕES}

De acordo com a análise visual, nota-se de um modo geral, que na filtragem passa-baixa (Figuras 1 e 2) ocorreu além da suavização da imagem a atenuação de altas frequências, que correspondem às transições abruptas (e.g. limite entre áreas iluminadas e sombreadas), tendendo a minimizar ruídos, e com isso, apresentando o efeito de borramento da imagem. Nelas observam-se duas regiões homogêneas, uma clara de floresta, e uma escura de solo exposto.

Na Figura 1, foi verificada que a técnica de filtragem linear testada na banda do infravermelho apresentou resultado satisfatório, quando confrontada com a filtragem na banda vermelho (Figura 2), já que apresenta um melhor detalhamento de áreas específicas da imagem. 


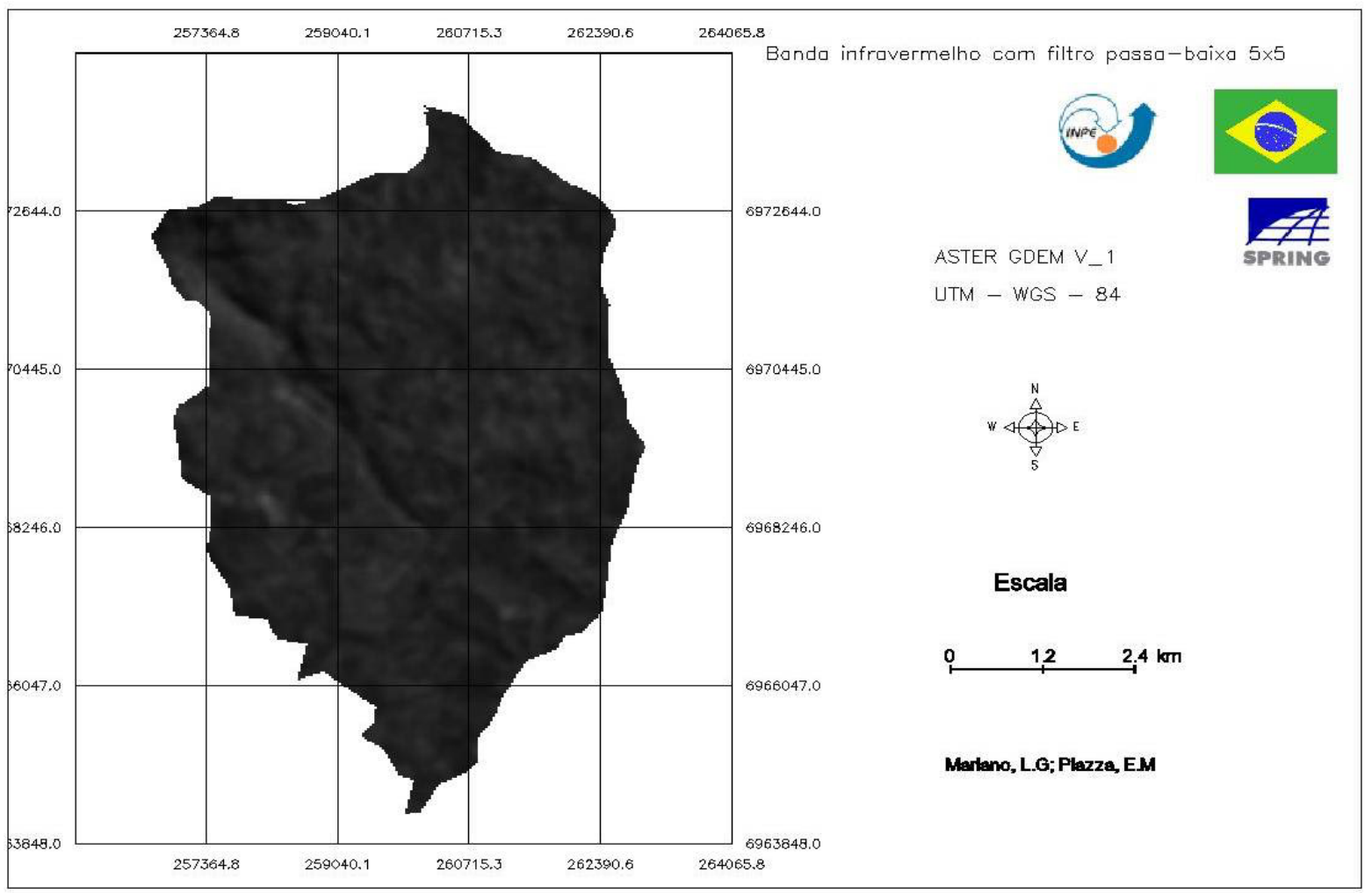

Figura 1. Imagem filtrada com a banda do infravermelho e filtro passa-baixa $5 \times 5$

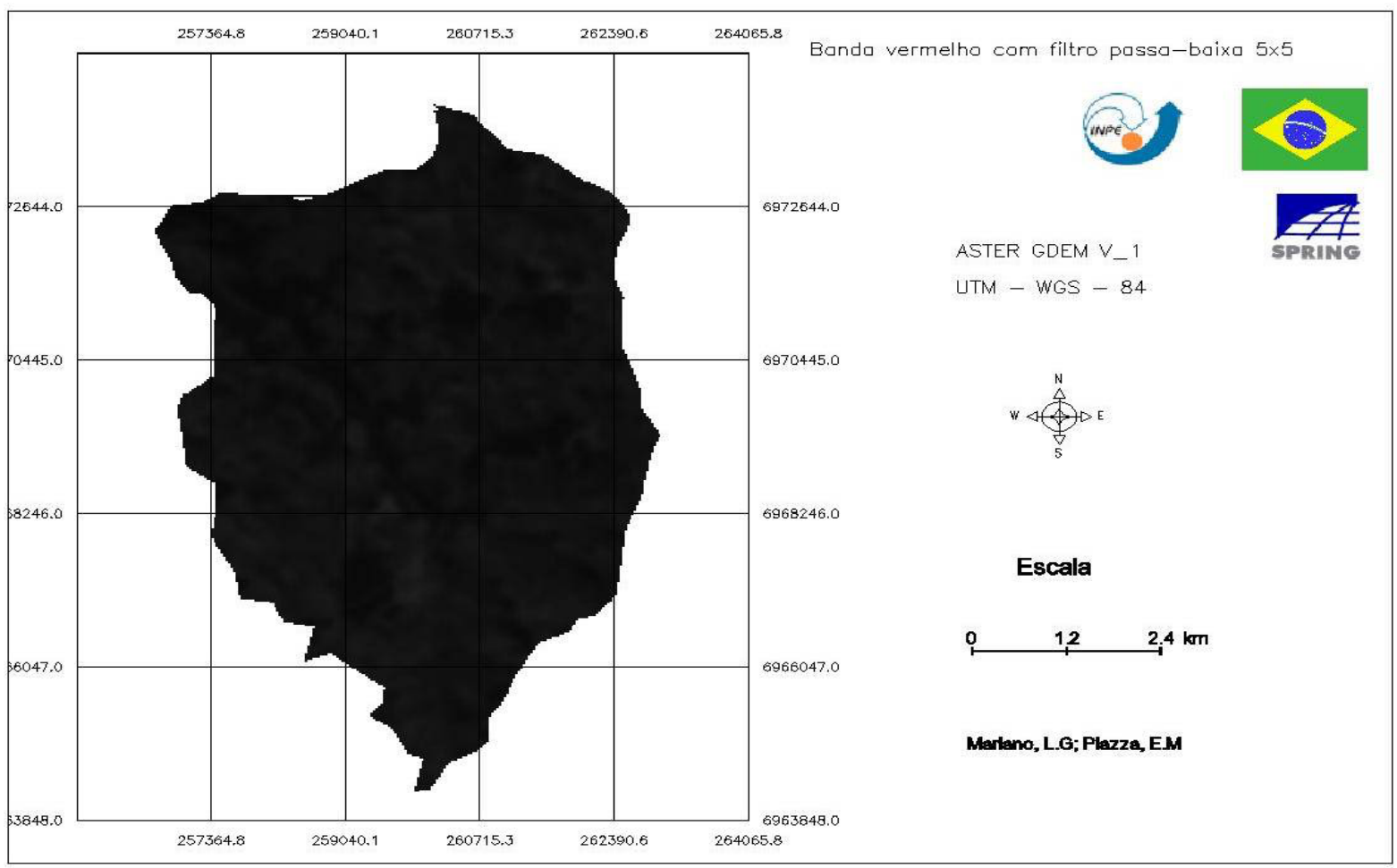

Figura 2. Imagem filtrada com a banda do vermelho e filtro passa-baixa $5 \times 5$ 


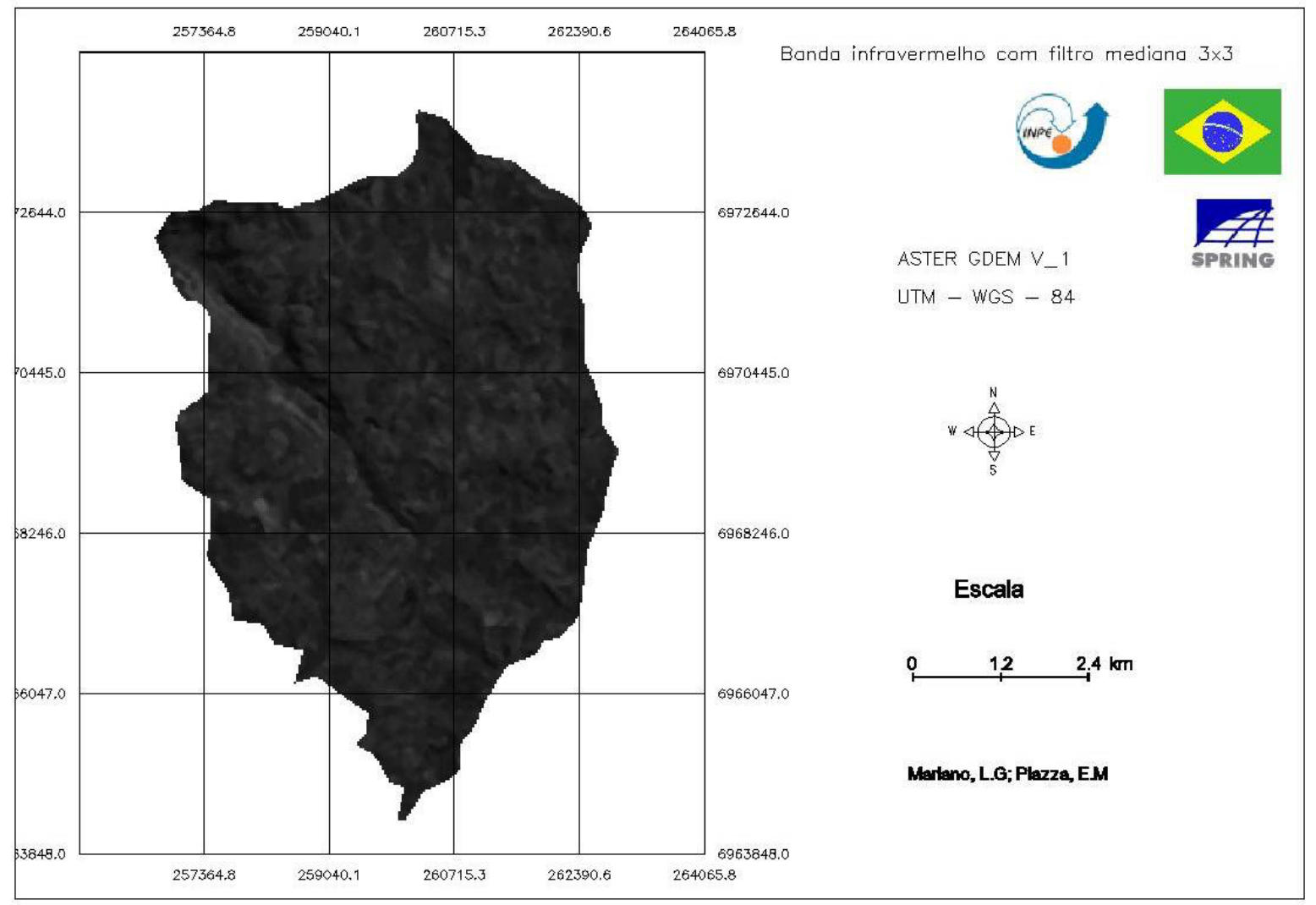

Figura 3. Imagem filtrada com a banda do infravermelho e filtro mediana $3 \times 3$.

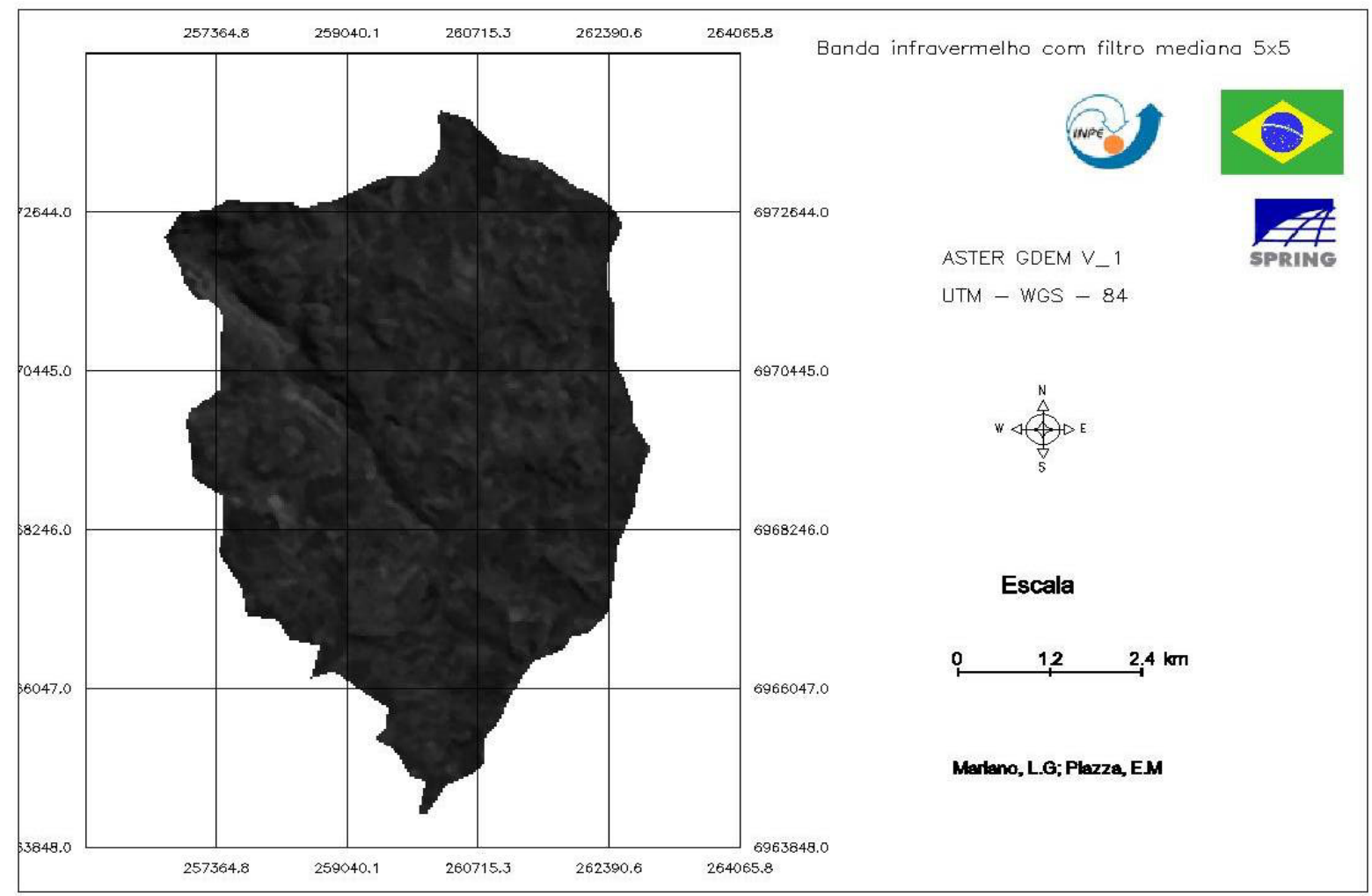

Figura 4. Imagem filtrada com a banda do infravermelho e filtro mediana $5 \times 5$ 


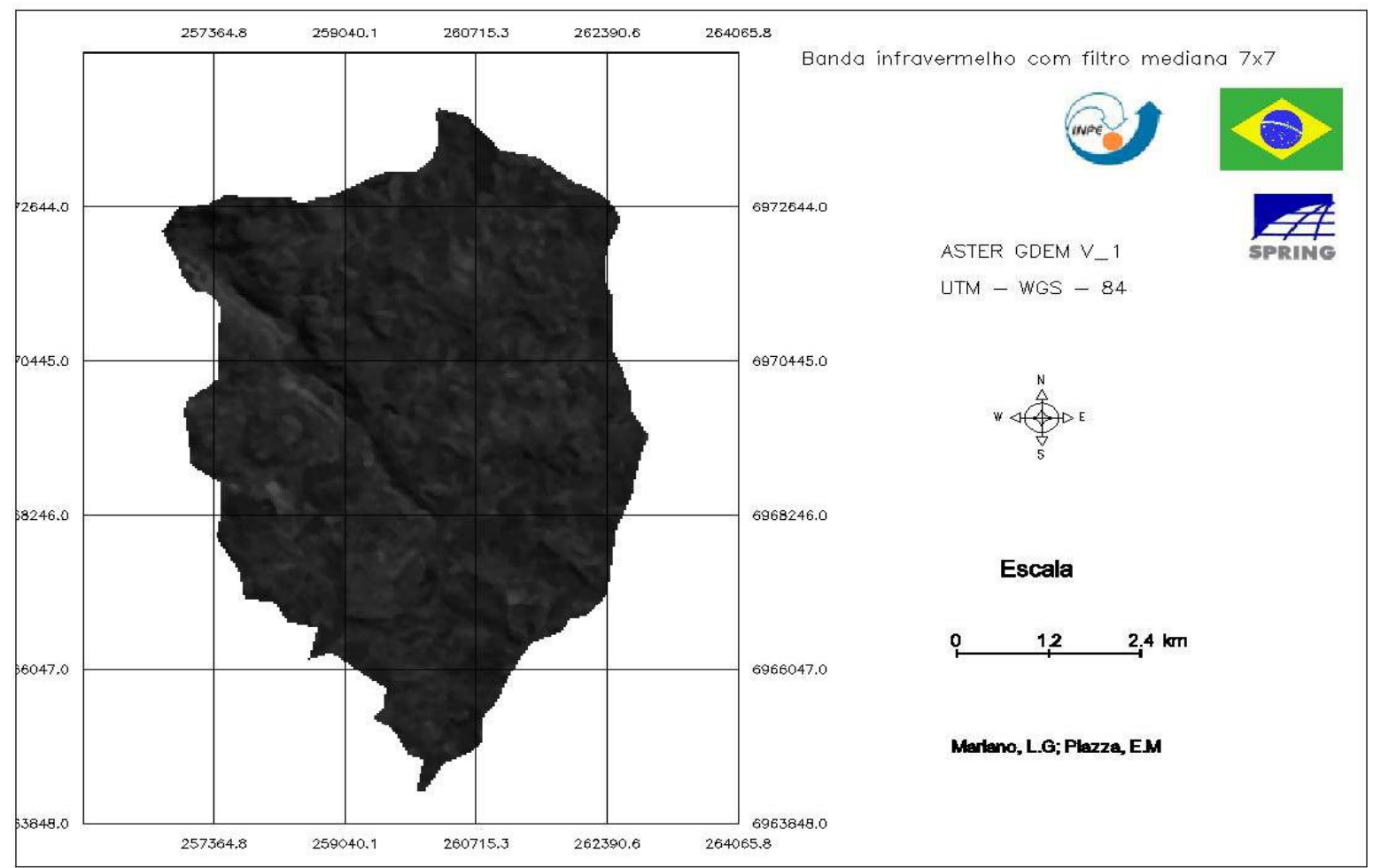

Figura 5. Imagem filtrada com a banda do infravermelho e filtro mediana $7 \times 7$.

Oliveira et al. (1984), afirma que na filtragem linear ocorre além do domínio do espaço através da operação de convolução, o domínio da frequência pela operação produto, suavizando e realçando detalhes da imagem e minimizando efeitos de ruído, sem alterar a média da imagem.

As Figuras 3, 4 e 5 são respectivamente o resultado de uma filtragem utilizando a média amostral. O filtro de mediana é o filtro mais simples para a redução do ruído, pois esta filtragem consiste em trocar o nível de cinza de um pixel pela media aritmética dos níveis de cinza de uma janela de tamanho $N \times N$ centrada neste pixel. Segundo Müller e Daronco (2000), os filtros de mediana são mais utilizados quando o objetivo for reduzir principalmente o borramento da imagem.

Observa-se que o melhor filtro, com relação ao critério de relação sinal-ruído é o da média, pois este filtro introduziu melhorias nas áreas claras e escuras. Cabe observar que a escolha do filtro mais adequado para determinado tipo de aplicação depende do objetivo da filtragem.

Outra observação importante diz respeito à avaliação qualitativa dos filtros, algumas destas formas são as estimações da perda da resolução através do desempenho dos métodos de classificação, detecção de bordas, análise de Fourier, e resposta impulsiva de um alvo pontual (SANT’ANNA, 1993).

\section{CONCLUSÃO}

Os processos de filtragem demonstram que a eficiência dos filtros varia de acordo com o alvo, alterando o contraste, brilho e estrutura. Desta forma, conclui-se que a utilização e comparação de filtros tende a colaborar para o entendimento da deterioração causada na imagem original, sendo extremamente importante buscar uma alternativa para comparação de filtros em pesquisas futurats.

\section{REFERÊNCIAS}

GONZALEZ, R. C., WOODS, R. E. Digital image processing. New York: Addison-Wesley Publishing Company, 1993.

MÜLLER, D. N.; DARONCO, E. L. Filtros espaciais passa-baixa. Disponível em: <http://www.inf. ufrgs.br/ danielnm/docs/FiltrosEspaciaisPassaBaixa.pdf>. Acesso em: 27 jul 2012.

OLIVEIRA, M.L.N.; FORESTI, C.; NIERO, M.; PARREIRAS, E.M.M.F. Estudo da evolução urbana de Brasília através do uso de dados LANDSAT. São José dos Campos: INPE, 1984. 26 p. 
SANT'ANNA, S. J. S. Avaliação de filttros redutores de Speckle. INPE: São José dos Campos. 1993.

SOLBO, S.; ELSOFT, T. A stationary waveletdomain wiener filter for correlated speckle. IEEE Transactions on Geoscience and Remote Sensing, v. 46, n. 04, p. 1219-1230, 2008. 